\title{
conf $781114--13$
}

MAGNETIC INTERACI:IONS IN TERNARY SUPERCONDUCTORS

\section{MASTER}

by

G. K. Shenoy, B. D. Dunlap, F. Y, Fradin, C, W. Kimba11,

W. Potze1, F, Probst and G. M. Kalvius

Prepared for

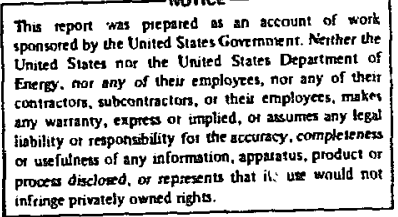

24 th Annual MMM Conference

Cleveland, Ohio

November $14-17,1978$

40

ARGONNE NATIONAL LABORATORY, ARGONNE, ILLINOIS

Operated under Contract W-31-109-Eng-38 for the

U. S. DEPARTMENT OF ENERGY 
Magnetic Interactions in Ternary Superconductors (Invited)

G. K. Shenoy, B. D. Dunlap, F. Y. Fradin, Argonne National Laboratory, Argonne, Illinois 60439*

C. W. Kimball, Northern Illinois University, DeKalb, Illinois $60115^{+}$

W. Potzel, F. Pröbst, G. M. Kalvius, Technical University of Munich, Garching, Germany

\section{ABSTRACT}

Numerous ternary superconductors containing rare-earth atoms have recently been studied in order to investigate the nagnetic interactions between the conduction electrons and local magnetic monents. These investigations range from the evaluation of the strength of spin-flip scattering of the conduction electrons off the rare-earth moments to the problem of the coexistence of superconductivity and gagnetic order. We present results of our studies on $(\mathrm{Eu}, \mathrm{Sn}) \mathrm{M}_{6} \mathrm{~S}_{8}, \mathrm{ErRh}_{4} \mathrm{~B}_{4}$, $\mathrm{Er}_{1-\mathrm{x}} \mathrm{Ho}_{\mathrm{x}} \mathrm{Rh}_{4} \mathrm{~B}_{4}$ and $\mathrm{Er}_{76} \mathrm{Gd}_{\mathrm{x}} \mathrm{Rh}_{4} \mathrm{~B}_{4}$ using primarily the uossbauer effect in $15 \mathrm{I}_{\mathrm{Eu}}$ and $16 \mathrm{E}_{\mathrm{Er}}$, and nuclear magnetic resonance of $95_{\mathrm{Ho}}$. The paramagnetic hyperfine spectra of the rare-earth ions indicate a weak coupling of the $4 f$ magnetic monent to the conduction electrons. The enhanced $\mathrm{H}_{\mathrm{c} 2}$ value in $(\mathrm{Eu}, \mathrm{Sn}) \mathrm{Mo}_{6} \mathrm{~S}_{8}$ is found to be related to a negative s-band polarization at the No site. The magnetic moment on the Er atom in $\mathrm{ErRh}_{4} \mathrm{~B}_{4}$ (and in related materials) in the nagnetically ordered state is found to be about $8.3 \mu_{B}$. This is $30 \%$ larger than that measured from neutron diffraction studies, indicating that only a component of tive moment shows long-range nagratic order.

PACS numbers: 74.70.Lp, 74.70.2s, 75.30.Hx. 


\section{INTRODUCTION}

The discovery of ternary materials which are superconducting while at the same time containing large concentrations of rare-earth (RE) atoms $[1,2]$ (e.g., $\mathrm{RFMl}_{6} \mathrm{~S}_{8}, \mathrm{REMO}_{6} \mathrm{Se}_{8}$ and $\mathrm{RERh}_{4} \mathrm{~B}_{4}$ ) has presented new opportunities for exploring questions related to the interaction between magnet ism and superconductivity $[3,4]$. As a result of various investigations of these materials, a number of important questions have arisen. Some of these are listed below.

(1) In these materials there is a large concentration ( $15 \%$ ) of magnetic atons. Why is superconductivity not destroyed by the treaking of Cooper pairs (spin depairing) due to the exchange interaction between conduction electron and rare-earth magnetic moments?

(2) The critical field $\left(\mathrm{H}_{\mathrm{C} 2}\right.$ ) values of $\mathrm{SnM}_{6} \mathrm{~S}_{8}$ (and related compounds) are quite large ( $300 \mathrm{kG})$. Partially replacing $\mathrm{Sn}$ atoms by paranagnetic Eu2t atoms increases $\mathrm{H}_{\mathrm{c}} 2$ without depressing the superconducting transition temperature $[1,5]$. What is the rechanism by which magnetic inurities enhance superconducting properties?

(3) $\operatorname{ErRh}_{4} \mathrm{P}_{4}$ becomes superconducting at $8.5 \mathrm{~K}$. On lowering the temperature to $0.9 \mathrm{~K}$, the superconduccivity is destroyed [6] and long-range aagnetic ordering is observed [7]. What is the nature of the magnetically ordered state?

In the following we will review recent work which has addressed these questions. Measurenents of magnetic interactions, degree of magnetic order, etc. as obtained from microscopic tools such as the Mossbauer effect, NRR and neutron scattering will be discussed.

\section{SPIN DEPAIRING INTERACTIONS}

It is well known that paramagnetic inpurities in superconducting materials will depress the superconducting properties. This arises from an exchange interaction between the magnetic impurity and the conduction electron spin which breaks up the superconducting electron pairs. The theory aeveloped by Abrikosov and Gor ${ }^{\circ}$ kov [8] shows that the depression in $T_{c}$ is related to the product of the density of states at the Fermi surface $N\left(E_{F}\right)$ and the exchange coupling constant $J$. The validity of this theory has been tested in many superconducting materials [4]. However, the suppression of $\mathrm{T}_{c}$ is much less pro-nounced in the ternary superconductors than in other compounds. As an example, Fig. 1 shows [5] $T_{c}$ values in the system $\mathrm{Sn}_{1.2(1-\mathrm{x})} \mathrm{Eu}_{\mathrm{x}}{ }^{\mathrm{M} \mathrm{O}_{6}}{ }_{6.25} \mathrm{~S}_{8}$. As $\mathrm{x}$ increases 
up to about $0.5, T_{c}$ is hardly changed, the deprassion finally occurring only at very high concentrations. It is thus of interest to determine the strength of the depairing interaction in the ternary compounds. In order to measure the exchange coupling between the Eu spin and the conduction electron spins, we

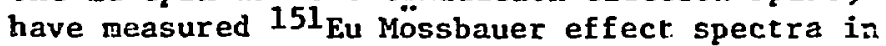

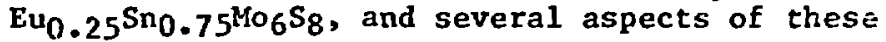
measurements will now be discussed.

The value of the Mössbauer 1somer shift reflects the total electron density at the nucleus. The measured value of $-14.0 \pm 0.1 \mathrm{~mm} / \mathrm{s}$ in this compound

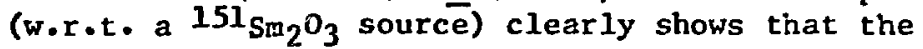
Eu atom is divalent and hence has a large magnetic moment of $7 \mu_{B}$. From the systematics of $151_{E u}$ isoner shifts, this value is found to be typlcal for Eu2t in an ionic compound. In Eu2t intermetallics, isoner shifts of about half this value are nore common [9]. For example, in superconducting $\mathrm{Eu}_{1.2} \mathrm{La}_{98.5}$

the shift is about $-8 \mathrm{~mm} / \mathrm{s}[10]$. Thus in an atonic picture, the 6s density at the magnetic Eu site in the Eu-La alloys is considerably higher than in $(\mathrm{Eu}, \mathrm{Sn}) \mathrm{Mo}_{6} \mathrm{~S}_{8} \cdot$ One may therefore conclude that the magnetic Eu atoms are fairly isolated fron the conduction electrons in $(\mathrm{Eu}, \mathrm{Sn}) \mathrm{Mo}_{6} \mathrm{~S}_{8}$. The direct measure of the depairing interaction is the spin relaxation rate of the Eu spin as a function of temperature. This relaxation rate, if dominated by exchange scattering from the conduction electrons (Korringa process), can be written

$$
V=\frac{2 \pi}{\hbar}\left(g_{J}-1\right)^{2}\left[J N\left(E_{F}\right)\right]^{2} \mathrm{kT}
$$

In the case where the relaxation rate is large compared with nuclear Larmor frequencies, this will produce an excess linewidth $\Delta \Gamma$ proportional to the relaxation tIme [11]. From Ref. 11, one can show

$$
V\left(\mathrm{sec}^{-1}\right)=2.75 \times 10^{9} / \Delta \Gamma(\mathrm{mm} / \mathrm{sec})
$$

for this case. The observed temperature dependence of the relaxation rate is shown in Fig. 2. In the normal state, the Korringa relaxation is obeyed and we obtain $\left|J N\left(E_{F}\right)\right|=0.0077$ states/aton spin. Below the transition temperature of $T_{c}=12 \mathrm{~K}$, the relaxation rate is found to be larger than would be given by an extrapolation of the Korringa law. However, this may simply represent the Increasing importance of relaxation due to dipolar interactions among Eu spins at these low temperatures. 
The important result is that $\operatorname{Ji}\left(E_{F}\right)$ is substantially smaller than that for $F u$ in any other conducting system. For example, in $\mathrm{LaAl}_{2}: \mathrm{Eu}$, it is 0.05 states/atom spin [12]. One thus obtains a direct observation of a small density of states at the Eu site and/or a sinall exchange coupling. This in turn is in agreement with the weak. dependence of $T_{c}$ on Eu concentration for $x \leq 0.5$. In order. to obtain a value of $\mathrm{J}$, a calculation of the density of states and its $\ell$-decomposition projected on the magnetic sites (such as was recently obtained for $\operatorname{ErRh}_{4} B_{4}$ [13]) will be necessary. At higher concentrations, short-range magnetic ordering appears to be present and at very low temperatures ( $0.5 \mathrm{~K}$ ) something comparable to a spin-glass state occurs [14]. This may be related to the eventual decrease in $T_{C}$, since one knows that $T_{c}$ can be depressed when the nagnetic correlation length becones comparable with the superconducting coherence length [15].

\section{ENHANCERENT OF THE UPPER CRITICAL FIELD} ...

In Fig. 1, we show the variation of the upper critical field with concentration fin the systen $\mathrm{Sn}_{1.2(1-\mathrm{x})} \mathrm{Eu}_{\mathrm{x}} \mathrm{Ho}_{6.35} \mathrm{~S}_{8}$. One sees that the presence of the paramagnetic inpurity can actually enhince the superconducting properties. Such an enhancenent was suggested by Jaccarino and Peter [16] as a possibility if the paramagnetic ion induced a conduction electron polarization with a sign that would partically compensate the applied field. The critical field can be described through the following expression [5]

$$
\mathrm{H}_{\mathrm{c} 2}(\mathrm{~T}, \mathrm{X})=\mathrm{H}_{\mathrm{c} 2}^{*}(\mathrm{~T}, \mathrm{x})-0.022 \frac{\alpha}{\lambda_{\mathrm{so}} \mathrm{T}_{\mathrm{c}}}\left[\mathrm{H}_{\mathrm{c} 2}(\mathrm{~T}, \mathrm{x})+\mathrm{H}_{\mathrm{J}}(\mathrm{x})\right]^{2}
$$

Fere $\mathrm{H}_{\mathrm{c} 2}^{*}$ is the orbital critical field and the second tern describes the reduction in the critical field due to the paramagnetic depairing. $\alpha$ is the Maki parameter and $\lambda_{\text {so }}$ is the spin-orbit coupling. The second term also includes the exchange field $\mathrm{H}_{\mathrm{J}}$ acting on the conduction electron spins, which is proportional to $x$. If $H_{J}>0, H_{c 2}$ will decrease with increasing $x$. However, if $\mathrm{H}_{\mathrm{J}}<0$ and $\mathrm{H}_{\mathrm{c} 2}^{*}(\mathrm{~T}, \mathrm{x})$ has a weak (or no) dependence on $x$ compared to $\mathrm{H}_{\mathrm{J}}(\mathrm{x})$, then $H_{s_{2}}$ will increase with $x$. A near independence of $\mathrm{H}_{\mathrm{c} 2}$ on $\mathrm{x}$ is, in fact, anticipated in this system because of the weak exchange interaction between rare-earth atoms and the conduction electrons, as indicated by the weak depression of $\mathrm{T}_{\mathrm{c}}$ • 
We have investigated this question by loo:ing for conduction eleetron spin polarization contributions to the hyperfine field in $\mathrm{Sn}_{0.5} \mathrm{Eu}_{0.5} 5_{95} \mathrm{Ho}_{8}$, using the $151_{\mathrm{Eu}}$ lössbiquer resonance and the 950 Knight shift [17]. The results of this investigation show that the spin-polarization is positive at the Eu site, but has a spatial variation such that it is negative at the Mo site. Since it is presumed that the superconductivity arises from the Mo d-electrons, the negative spin-polarization will provide the compensation discussed above. This, therefore, provides a direct observation of the origin of the critical field enhancement.

The details of these measurements lead to a two band scheme for $\mathrm{Eu}_{0 .} \mathrm{Sn}_{0.5} \mathrm{Mo}_{6} \mathrm{~S}_{8}$. In Fig. 3 we Indicate an "s-band" and a "d-band". It is presumed that the superconducting properties are due to the $d$ band, derived largely from the No ions. Below $T_{c}$, it is presumed that a gap opens in the $d$ band, but not in the $s$ band. This is supported by the 95 Mo measureqents. The Knight shift is made up of a s-electron (contact interaction) part, $K_{s}$, and a d-electron (core polarization) part $K_{d}$. The second part should show no gross temperature dependence except that it will disappear below $T_{c}$ due to pairing of the $d$ electrons; this is seen both for $\mathrm{SnHo}_{6} \mathrm{~S}_{8}$ and

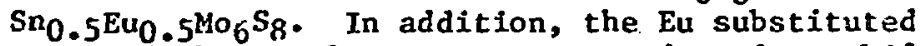
compound shows a large temperature dependent sint which is not seen in pure $\mathrm{SnMo}_{6} \mathrm{~S}_{8}$. This dependence is found to be proportional to the bulk magnetization and so arises from spin polarization due to exchange between the rare-earth and conduction electrons. This shift does not disappear below $T_{c}$ due to the fallure of the $s$ band to participate in the superconductivity. Hence the principal effect of the Eu impurities initially is to polarize the s-band and provide the $\mathrm{H}_{\mathrm{c} 2}$ compensation effect, while the weak s-d coupling prevents a severe attenuation of $T_{c}$.

\section{SUPERCONDUCTIVITY AND ORDERED MAGNETISA}

There now exist a large number of experiments that deal with the question of co-existence between superconductivity and ordered magnetism. These can be divided into three classes: (1) The bulk of the work has dealt with pseudobinary compounds, largely of C15 cubic Laves phases. It is presentiy believed that most of these systens do not show long-range magnetic order, but do exhibit either short-range order or something like spin-glass behavior [18]. In many of these cases, there exists regions in the phase diagram where this ordering is co-existent with superconductivity. (2) Ii some ternary coupounds, 
$\left(\mathrm{e} . \mathrm{g}^{\circ}, \mathrm{Dy}_{1.2} \mathrm{Mo}_{6} \mathrm{~S}_{8}\right)$, true coexistence has been found between superconductivity and long-range anti-ferromagnetic order [19] - (3) Other ternary compounds exhibit re-entrant behavior where long-range magnetic order develops and, as a result, the superconductivity is destroyed. Examples of this behavior are seen in $\mathrm{Ho}_{1.2} \mathrm{Mo}_{6} \mathrm{~S}_{8}[20]$ and $\mathrm{ErRh}_{4} \mathrm{~B}_{4}[1,6]$. In both of these cases the ordering is ferronagnetic. In this section we will present results of Mossbauer studies on $\operatorname{ErRh}_{4} B_{4}$ in the superconducting and nagnetic states. These results show that the Er magnetic moment is not fully ordered even in the magnetic state [21].

In Fig. 4 we show the Mossbauer spectra of ErRh $_{4} B_{4}$ measured with the $80.6 \mathrm{keV}$ resonance of $1_{\mathrm{Er}}$ in $\mathrm{ErRh}_{4} \mathrm{~B}_{4}$ at $1.5 \mathrm{~K}$ and $0.1 \mathrm{~K}$. The first is below the superconducting transition $\left(T_{c}=8.5 \mathrm{~K}\right.$ ) but above the magnetic transition $\left(T_{M}=0.93 \mathrm{~K}\right)$ and the second is below $T_{M}$. In the superconducting state, a fully split hyperfine pattern is seen due to the fact that the electronic spin relaxation rate of the Er atom is small compared to nuclear Larmor frequencies. In the magnetically ordered state, the spectrum is essentially identical except for some decreases in the linewidths of the different hyperfine components.

From the measured hyperfine field we can obtain a value for the magnetic moment on the $\mathrm{Er}^{3+}$ atom. The hyperfine field is given by

$$
H_{n}=2 \mu_{B}\left\langle r^{-3}\right\rangle_{4 f}\langle J|| N|| J\rangle\left\langle J_{z}\right\rangle_{T}+\frac{8 \pi}{3} s_{e} \delta(r)
$$

The second term on the right is the contact interaction, made up of a core polarization contribution [22] and the conduction electron contribution both of which are negligible in this case. The first term represents the orbital hyperfine field. Here, $\langle\mathrm{J}|| \mathrm{N}|| \mathrm{J}\rangle$ is an appropriate reduced matrix element, $\left\langle\mathrm{r}^{-3_{3}}\right\rangle_{4 \mathrm{f}}$ is a radial average for $4 f$ electrons, and $\left\langle J_{z}\right\rangle_{T}$ is the thermal average of the angular monentum over all crystalline elecric field (CEF) levels. One sees that both $H_{n}$ and the magnetic moment $\mu_{\text {are proportional }}$ to $\left\langle J_{2}\right\rangle_{T}$. The resultant proportionality between $H_{n}$ and $\mu$ is shown for a large number of $\mathrm{Er}^{3+}$ systens in Fig. 5. From the measured hyperfine field at 0.1 $K$ in $\operatorname{ErRh}_{4} B_{4}$, we obtain a moment of $(8.3 \pm 0.2) \mu_{B}$ on the Er atom. This should be compared with the neutron diffraction result of $5.6 \mu_{B}$ in the ordered state [7]. In order to reconcile the Mossbauer and neutron results, it appears that we must have a situation where only a component of the magnetic moment ( $5.6 \mu_{B}$ in magnitude) is ordered, with the other components belng disordered in either a statie 
or dynamic fashion. Since the neutron diffraction technigue measures a long-range spin correlation. it will not observe the disordered component. However, the Mössbauer technique measures a single-ion property (auto-correlation) and in the limit of slow electronic spin relaxation will measure the full moment. It will be of considerable interest to find if such a disorder can be reduced by the application of an external field.

Nuch attention in the past has been paid to the disruptive influence of magnetic spins on the superconducting state. We would like to point out that the role of superconducting interactions in the ordered magnetic state may be equally important. In a system with two coupled order parameters (superconducting and nagnetic) which have opposing effects on the conduction electrons, the system may obtain a minimum free-energy by maintaining sone disorder in the magnetic moment. Thus, although we expect long-range ordering of the entire motent on the $\mathrm{Er}$ atoms at $0.1 \mathrm{~K}\left(\sim \mathrm{T}_{\mathrm{M}} / 10\right)$, only a portion of the moment is ordered. The suggestion that superconducting correlations are still influential below $T_{M}$ is supported by the resitivity of $\mathrm{ErRh}_{4} \mathrm{~B}_{4}$ in the magnetic state relative to that in the normal state (induced either by external field or temperature) [6]. The Mössbauer measurements on $166_{\mathrm{Er}}$ in numerous pseudo-ternary alloys of the type $\left(\mathrm{Er}_{1-\mathrm{x}} \mathrm{Gd}_{\mathrm{x}}\right) \mathrm{Rh}_{4} \mathrm{~B}_{4}$ and $\left(\mathrm{Er}_{1-\mathrm{x}} \mathrm{Ho}_{\mathrm{x}}\right) \mathrm{Rh}_{4} \mathrm{~B}_{4}$ in their magnetically ordered, superconducting and normal states yield approximately the same sized magnetic moment on the Er aton [23]. This establishes the generality of sone of the results discussed above.

\section{CONCLUSTONS}

We have reviewed here recent Mössbauer effect and NWR work on magnetic interactions in relation to other properties of ternary superconductors. In the Chevrel phase compounds $(\mathrm{Eu}, \mathrm{Sn}) \mathrm{Mo}_{6} \mathrm{~S}_{8}$ we have suggested a two band plcture in which Fu spins are only weakly coupled to the "s-band" electron, and does not disturb the "d-band" superconductivity. The "s-band" polarization at the No sites, in the presence of $\mathrm{Eu}$ atons, shields the externally applied field, thus enhancing the $\mathrm{H}_{\mathrm{c} 2}$ values.

The re-entrant superconductor $\operatorname{ErRh}_{4} \mathrm{~B}_{4}$ has an unusual magnetic order in which only a component of the total Er moment shows long-range ferronagnetic correlation, with the remaining components being disordered down to $0.1 \mathrm{~K}$.

At present, relatively fow experiments have been performed utilizing microscopic tools to study magnetic interactions in superconductors. There is 
now an established place for work of this nature, and many results nay be expected in the near future. Commensurately, there is a need for theoretical studies of the interaction between nagnetic and superconducting order parareters, including the possiblity of a combined superconducting-nagnetic ground state.

We wish to thank A. T. Aldred, A. J. Freeman, G. H. Lander and S. K. Sinha for nurerous discussions.

\section{REFERENCES}

* Work supported by the U. S. Department of Energy.

+ Work sponsored by the National Science Foundation.

1. $\emptyset$. Fischer, Appl. Phys. 16, 1 (1978).

2. M. B. Maple, 15th LT Conference, Grenoble, France, Aug. 1978. J. Phys. (Paris), in press,

3. $\emptyset$. Fischer and $M$. Peter in Magnetism, Vol. 5. Ed. H. Suhl (A.cademic Press, New York), p. 327.

4. M. B. Maple, App1. Phys. 9, 179 (1976).

5. $\emptyset$. Fischer, M. Decroux, S. Koth, R. Chevrel, and $M$. Sergent, J. Phys. CE, L474 (1975).

6. W. A. Feriig, D. C. Johnston, L. E. Delong, R. W. McCallum, M. B. Maple, and B. T. Matthias, Phys. Rev. Letters 38, 987 (1977).

7. D. E. Moncton, D. B. McWhan, J. Eckert, G. Shirane, and W. Thomlinson, Phys. Rev. Letters 39, 1164 (1977).

8. A. A. Abrikosov and L. P. Gor'kov, Sov. Phys. JETP 12, 1243 (1961).

9. E. R. Bauninger, G. M. Kalvius, and I, Nowik, in Mossbauer Isomer Shifts, Eds. G. K. Shenoy and F. E. Wagner (North Holland Publ. Co., Ansterdam, 1978), p. 694.

10. P. Steiner, D. Gumprecht, and S. Hufner, Phys. Rev. Letters 30, 1132 (1973).

11. E. Bradford and W. Marshall, Proc. Phys. Soc. (London) 87, 731 (1966).

12. G. Koopman, U. Engel, K. Baberschke, and S. Hüfner, Sol. State Conmun. 11, 1197 (1972)

13. J. Jarlborg, A. J. Freenan, T. J. Watson-Yang Phys. Rev. Letters 39, 1032 (1977)

14. J. Bolz, G. Crecelius, H. Maletta, F. Pobell,

J. Low Temp. Phys. 28, 611 (1977)

15. P. W. Anderson and H. Suhl, Phys. Rev. 126, 898 (1959)

16. V. Jaccarino and $\mathrm{M}$. Peter, Phys. Rev. Letters 9, 290 (1962).

17. F. Y. Fradin, G. K. Shenoy, B. D. Dunlap, A. T. Aldred, and C. W. Kimball, Phys. Rev. Let ters 28, 719 (1977).

18. For a review of these studies sea S. Roth, Appl. Phys. 15, 1 (1978). 
19. M. Ishikawa and $\emptyset$. Fischer, Solid State Commun. 24, 747 (1977); D. E. Moncton, G. Shirane, W. Thomlinson, M. Ishikawa, and $\emptyset$. Fischer, Phys. Rev. Letters 41, 1133 (1978)

20. M. Ishikawa and $\emptyset$. Fischer, Solid State Connun. 23, 37 (1977).

21. G. K. Shenoy, B. D. Dunlap, F. Y. Fradin, S. K. Sinha, C. W. Kimbal1, W. Potzel, F. Pröbst, and G. M. Kalvius, to be published.

22. K. E. Watson, P. Bagus, and A. J. Freeman, Bul1. An. Phys. Soc. 13, 482 (1968).

23. G. K. Shenoy, B. D. Dunlap, F. Y. Fradin, and C. W. Kimball, Bull. Am. Phys. Soc. 23, 306 (1978) 
Fig. 1. Concentration dependence of the critical field $\mathrm{H}_{c 2}$ (solid circles) and transition temperature $\mathrm{T}_{c}$

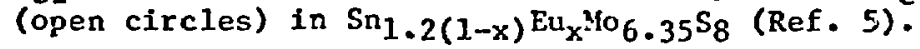

Fig. 2. Temperature dependence of the electronic relaxation rate for $\mathrm{Eu}$ in $\mathrm{Eu}_{0.25} \mathrm{Sn}_{0.75} \mathrm{Mo}_{6} \mathrm{~S}_{8}$ -

Fig. 3. Tro band picture for $\mathrm{SnMo}_{\epsilon} \mathrm{S}_{8}$ showing (a) conduction bands in the normal state and (b) the energy gap $E_{g}$ of the "d-band" in the superconducting state. The gap $E_{\mathcal{G}}$ is exaggerated in size. Thernally excited electrons are above the gap in the $d$-band and above $\mathrm{E}_{\mathrm{F}}$ in the $\mathrm{s}$ band for $\mathrm{T} \leq \mathrm{T}_{\mathrm{c}}$.

Fig. 4. Mossbauer spectra for ${ }^{166} \mathrm{Er}$ in $\operatorname{ErRh}_{4} \mathrm{~B}_{4}$ for. (a) the superconducting state and (b) the magnetically ordered state.

Fig. 5. Linear dependence of the Er magnetic hyperfine field on the electronic magnetic moment in various compounds. 